\title{
Homoclinics for singular strong force Lagrangian systems in $\mathbb{R}^{N}$
}

\author{
Marek Izydorek ${ }^{1}$ • Joanna Janczewska ${ }^{1} \cdot$ Nils Waterstraat $^{2}$
}

Received: 30 October 2019 / Accepted: 31 December 2020 / Published online: 5 April 2021

(c) The Author(s) 2021

\section{Abstract}

We will be concerned with the existence of homoclinics for Lagrangian systems in $\mathbb{R}^{N}$ $(N \geq 3)$ of the form $\frac{d}{d t}(\nabla \Phi(\dot{u}(t)))+\nabla_{u} V(t, u(t))=0$, where $t \in \mathbb{R}, \Phi: \mathbb{R}^{N} \rightarrow[0, \infty)$ is a $G$-function in the sense of Trudinger, $V: \mathbb{R} \times\left(\mathbb{R}^{N} \backslash\{\xi\}\right) \rightarrow \mathbb{R}$ is a $C^{2}$-smooth potential with a single well of infinite depth at a point $\xi \in \mathbb{R}^{N} \backslash\{0\}$ and a unique strict global maximum 0 at the origin. Under a strong force type condition around the singular point $\xi$, we prove the existence of a homoclinic solution $u: \mathbb{R} \rightarrow \mathbb{R}^{N} \backslash\{\xi\}$ via minimization of an action integral.

Mathematics Subject Classification Primary 37J45 - 46E30; Secondary 34C37 · 70H05

\section{Introduction}

Tanaka showed in [28] the existence of homoclinic solutions for singular Lagrangian systems in $\mathbb{R}^{N}, N \geq 3$, of the type

$$
\ddot{u}(t)+\nabla_{u} V(u(t))=0,
$$

where $V: \mathbb{R}^{N} \backslash\{\xi\} \rightarrow \mathbb{R}$ for some $\xi \neq 0$ has a unique global maximum at $0 \in \mathbb{R}^{N}$ and $|V(x)| \rightarrow \infty$ as $x \rightarrow \xi$. Roughly speaking, these homoclinic orbits were obtained by considering an approximate problem on a finite interval whose solutions are the critical points of a functional. Under suitable assumptions on the potential $V$, critical points of this functional can be obtained by a minimax argument, and they, as well as their minimax values,

Communicated by P. Rabinowitz.

$\bigotimes$ Joanna Janczewska

joanna.janczewska@pg.edu.pl

Marek Izydorek

marek.izydorek@pg.edu.pl

Nils Waterstraat

nils.waterstraat@mathematik.uni-halle.de

1 Faculty of Applied Physics and Mathematics, Gdańsk University of Technology, Narutowicza 11/12, 80-233 Gdańsk, Poland

2 Naturwissenschaftliche Fakultät II, Institut für Mathematik, Martin-Luther-Universität Halle-Wittenberg, 06099 Halle, Saale, Germany 
can be estimated uniformly in the length of the finite interval. This finally allows to construct a sequence converging weakly to a homoclinic orbit of (1). More precisely, his theorem reads as follows.

Theorem 1.1 Assume that

$\left(V_{a}\right) \quad V: \mathbb{R}^{N} \backslash\{\xi\} \rightarrow \mathbb{R}$, where $\xi \in \mathbb{R}^{N} \backslash\{0\}$, is a $C^{2}$-smooth potential such that

$$
\lim _{x \rightarrow \xi} V(x)=-\infty
$$

$\left(V_{b}\right)$ for all $x \in \mathbb{R}^{N} \backslash\{0\}, V(x) \leq 0$ and $V(x)=0$ iff $x=0$,

$\left(V_{c}\right)$ there is a negative constant $V_{0}$ such that

$$
\limsup _{|x| \rightarrow \infty} V(x) \leq V_{0}
$$

$\left(V_{d}\right)$ there is a constant $\delta \in\left(0, \frac{1}{2}|\xi|\right)$ such that $V(x)+\frac{1}{2}\left(V^{\prime}(x), x\right) \leq 0$ for all $x \in B_{\delta}(0)$, where $B_{\delta}(0)=\left\{x \in \mathbb{R}^{N} ;|x|<\delta\right\}$,

$\left(V_{e}\right)$ there are a neighbourhood $\mathcal{N} \subset \mathbb{R}^{N}$ of the singular point $\xi$ and a function $U \in$ $C^{1}(\mathcal{N} \backslash\{\xi\}, \mathbb{R})$ such that $|U(x)| \rightarrow \infty$ as $x \rightarrow \xi$, and for all $x \in \mathcal{N} \backslash\{\xi\}$,

$$
|\nabla U(x)|^{2} \leq-V(x)
$$

Then (1) possessess at least one (nontrivial) solution such that

$$
u(t), \dot{u}(t) \rightarrow 0 \text { as } t \rightarrow \pm \infty .
$$

In this work we will be concerned with the more general problem of existence of solutions for the nonautonomous Lagrangian systems

$$
\left\{\begin{array}{l}
\frac{d}{d t}(\nabla \Phi(\dot{u}(t)))+\nabla_{u} V(t, u(t))=0 \\
\lim _{t \rightarrow \pm \infty} u(t)=\lim _{t \rightarrow \pm \infty} \dot{u}(t)=0,
\end{array}\right.
$$

where $t \in \mathbb{R}, \Phi: \mathbb{R}^{N} \rightarrow[0, \infty)$ is a $G$-function in the sense of Trudinger, and $V: \mathbb{R} \times$ $\left(\mathbb{R}^{N} \backslash\{\xi\}\right) \rightarrow \mathbb{R}$ is a $C^{2}$-smooth potential which is 1 -periodic in $t$ and has as in Tanaka's Theorem 1.1 a well of infinite depth at a point $\xi \in \mathbb{R}^{N} \backslash\{0\}$ and a strict global maximum 0 at the origin. Moreover, we extend several preliminary results in [17] to the case $N \geq 3$, which is of independent interest.

Let us first introduce the notation that is needed to state our main theorem. We assume that the $C^{1}$-function $\Phi: \mathbb{R}^{N} \rightarrow \mathbb{R}$ satisfies the following conditions:

(G1) $\Phi(0)=0$,

(G2) $\Phi$ is coercive, i.e. $\lim _{|x| \rightarrow \infty} \frac{\Phi(x)}{|x|}=\infty$,

(G3) $\Phi$ is convex, i.e. $\Phi(a x+(1-a) y) \leq a \Phi(x)+(1-a) \Phi(y)$ for each $a \in[0,1]$ and all $x, y \in \mathbb{R}^{N}$,

(G4) $\Phi$ is symmetric, i.e. $\Phi(x)=\Phi(-x)$ for all $x \in \mathbb{R}^{N}$,

(G5) $\nabla \Phi \in C^{1}\left(\mathbb{R}^{N} \backslash\{0\}, \mathbb{R}^{N}\right)$.

Note that every $\Phi$ that satisfies $(G 1)-(G 5)$ is a $G$-function in the sense of Trudinger (compare [29]), and moreover $\Phi(x) \geq 0$ for all $x \in \mathbb{R}^{N}$. 
The Fenchel transform $\Phi^{*}: \mathbb{R}^{N} \rightarrow \mathbb{R}$ of $\Phi$ is defined by

$$
\Phi^{*}(y)=\sup _{x \in \mathbb{R}^{N}}((x, y)-\Phi(x)),
$$

where $(\cdot, \cdot): \mathbb{R}^{N} \times \mathbb{R}^{N} \rightarrow \mathbb{R}$ is the standard inner product in $\mathbb{R}^{N}$ (cf. [21,27]). It is wellknown that $\Phi^{*}$ is continuous and satisfies $(G 1)-(G 4)$ (cf. [20]), as well as $\Phi^{* *}=\Phi$ (cf. [3]).

Throughout the paper we assume that $\Phi$ and $\Phi^{*}$ are globally $\Delta_{2}$-regular [19], i.e. there is a constant $L>0$ such that for each $x \in \mathbb{R}^{N}$

$$
\Phi(2 x) \leq L \Phi(x) \leq \frac{1}{2} \Phi(L x) .
$$

Applying the Mean Value Theorem to the convex function $\Phi$, we obtain the inequality

$$
\Phi(x)-\Phi(x-y) \leq(\nabla \Phi(x), y) \leq \Phi(x+y)-\Phi(x),
$$

which is satisfied for every $x$ and $y$ in $\mathbb{R}^{N}$. Setting $y=x$ and using the $\left(\Delta_{2}\right)$ property, this yields

$$
(\nabla \Phi(x), x) \leq \Phi(2 x) \leq L \Phi(x), \quad x \in \mathbb{R}^{N} .
$$

If a convex map is $C^{1}$, then its Fenchel transform is just the classical Legendre transform $[21, \S 2]$. Consequently,

$$
(\nabla \Phi(x), x)=\Phi(x)+\Phi^{*}(\nabla \Phi(x)), \quad x \in \mathbb{R}^{N},
$$

and we obtain from (4)

$$
\Phi^{*}(\nabla \Phi(x))=(\nabla \Phi(x), x)-\Phi(x) \leq(\nabla \Phi(x), x) \leq L \Phi(x), \quad x \in \mathbb{R}^{N} .
$$

We next want to derive from $\Phi$ a function $\varphi$ that is needed in the assumptions on the potential $V$ in our main theorem. Consider the function $\phi: \mathbb{R} \rightarrow \mathbb{R}$ defined by

$$
\phi(r)=\min \{\Phi(x):|x|=r\}
$$

and $\phi(-r)=\phi(r)$, where $|\cdot|: \mathbb{R}^{N} \rightarrow[0, \infty)$ is the standard norm. Let us recall that the epigraph of a function $f: \mathbb{R}^{N} \rightarrow \mathbb{R}$ is the set

$$
\text { epi }(f)=\left\{(x, t) \in \mathbb{R}^{N} \times \mathbb{R}: \quad f(x) \leq t\right\}
$$

(cf. [21]). As in [17], we define the supporting function $\varphi: \mathbb{R} \rightarrow \mathbb{R}$ for $\Phi$ by the formula

$$
\varphi=\operatorname{conv}(\phi),
$$

which means that epi $\varphi=\overline{\text { conv (epi } \phi)}$. Obviously,

$$
\Phi(x) \geq \varphi(|x|) \text { for } x \in \mathbb{R}^{N},
$$

and one can easily check that

- $\varphi$ is continuous and satisfies $(G 1)-(G 4)$;

- $\varphi$ satisfies the $\left(\Delta_{2}\right)$-condition, i.e. $\varphi$ and $\varphi^{*}$ are globally $\Delta_{2}$-regular, where $\varphi^{*}$ is defined as in (2).

Finally, let us consider the potential $V$ in $(L S)$, for which we will assume that 
$\left(V_{1}\right) \quad V: \mathbb{R} \times\left(\mathbb{R}^{N} \backslash\{\xi\}\right) \rightarrow \mathbb{R}$, where $\xi \in \mathbb{R}^{N} \backslash\{0\}$, is a $C^{2}$-function which is 1 -periodic in $t \in \mathbb{R}$ and such that

$$
\lim _{x \rightarrow \xi} V(t, x)=-\infty
$$

uniformly in the time variable $t$,

$\left(V_{2}\right)$ for all $t \in \mathbb{R}, x \in \mathbb{R}^{N} \backslash\{0\}, V(t, x)<V(t, 0)=0$ and $V^{\prime \prime}(t, 0)$ is negative definite,

$\left(V_{3}\right)$ there is a negative constant $V_{0}$ such that for all $t \in \mathbb{R}$,

$$
\limsup _{|x| \rightarrow \infty} V(t, x) \leq V_{0},
$$

$\left(V_{4}\right)$ there are a neighbourhood $\mathcal{N} \subset \mathbb{R}^{N}$ of the point $\xi$ and a function $U \in C^{1}(\mathcal{N} \backslash\{\xi\}, \mathbb{R})$ such that $|U(x)| \rightarrow \infty$ as $x \rightarrow \xi$, and for all $x \in \mathcal{N} \backslash\{\xi\}$ and $t \in \mathbb{R}$,

$$
\varphi^{*}(|\nabla U(x)|) \leq-V(t, x) .
$$

Let us note that it follows from $\left(V_{1}\right)$ and $\left(V_{2}\right)$ that

$\left(V_{*}\right)$ there is a constant $\delta \in\left(0, \frac{1}{2}|\xi|\right)$ such that $V(t, \lambda x) \geq \lambda V(t, x)$ for all $t \in \mathbb{R}, 0 \leq \lambda \leq 1$ and $x \in B_{\delta}(0)$.

We are now ready to state the announced strengthening of Theorem 1.1.

Theorem 1.2 Let $\Phi: \mathbb{R}^{N} \rightarrow[0, \infty)$ satisfy $(G 1)-(G 5)$ and $\left(\Delta_{2}\right)$, and assume that the potential $V: \mathbb{R} \times\left(\mathbb{R}^{N} \backslash\{\xi\}\right) \rightarrow \mathbb{R}$ satisfies $\left(V_{1}\right)-\left(V_{4}\right)$. Then there exists at least one nontrivial homoclinic solution of the problem $(L S)$ that begins and ends at 0.

Let us emphasize that we mean by solution an absolutely continuous function in $L^{2}\left(\mathbb{R}, \mathbb{R}^{N}\right)$ that satisfies $(L S)$ weakly and such that the function as well as its first derivative converge to 0 as $t \rightarrow \pm \infty$. Thus the reader might be tempted to believe that our result is weaker than Tanaka's Theorem 1.1. This, however, is not the case as the regularity of the obtained solution in Theorem 1.2 improves if $\Phi$ satisfies common additional assumptions. If we require in $(G 3)$ that $\Phi$ is not only convex but strictly convex, then the solution has a classical first derivative. Moreover, in the latter case we even obtain a classical solution of $(L S)$ if $\Phi^{*} \in C^{2}\left(\mathbb{R}^{N}, \mathbb{R}\right)$. We shall explain these facts in our final remarks in Sect. 4.

There are various important examples of functions $\Phi$ which satisfy $(G 1)-(G 5)$. Firstly, if we substitute $\Phi(x)=\frac{1}{2}|x|^{2}, x \in \mathbb{R}^{N}$, into $(L S)$, then we get (1). Moreover, we obtain the $p$-Laplacian for $\Phi(x)=\frac{1}{p}|x|^{p}, x \in \mathbb{R}^{N}, p>1$, as

$$
\frac{d}{d t}(\nabla \Phi(\dot{u}(t)))=\frac{d}{d t}\left(|\dot{u}(t)|^{p-2} \dot{u}(t)\right) .
$$

Note that it follows from the above comments that we obtain from Theorem 1.2 classical solutions in these cases as long as $1<p \leq 2$. Finally, for $\Phi(x)=\chi(|x|)$, where $\chi: \mathbb{R} \rightarrow \mathbb{R}$ is a so-called $N$-function (i.e. a $G$-function of one variable which satisfies additional growth conditions, cf. [4]), we obtain a $\chi$-Laplacian.

If we compare our assumptions on the potential $V$ in Theorem 1.2 to those in Theorem 1.1, we firstly see that $\left(V_{a}\right)$ and $\left(V_{c}\right)$ are special cases of $\left(V_{1}\right)$ and $\left(V_{3}\right)$. Moreover, in case that $\Phi(x)=\frac{1}{2}|x|^{2}, x \in \mathbb{R}^{N}$, i.e. if (1) is just ( $\left.L S\right)$ with an autonomous potential, it is readily seen that $\left(V_{e}\right)$ follows from $\left(V_{4}\right)$. Note that $\left(V_{e}\right)$ was introduced by Gordon [12] and in the literature it is known as the strong force condition or Gordon's condition. Finally, Tanaka states in [28] that $\left(V_{d}\right)$ is a kind of concavity condition near 0 . We have instead $\left(V_{*}\right)$, which 
follows from our assumptions $\left(V_{1}\right)$ and $\left(V_{2}\right)$. Note that $\left(V_{2}\right)$ is slightly more restrictive than $\left(V_{b}\right)$ as Tanaka allows an inconclusive second derivative test at the strict global maximum 0 .

In the last three decades, a lot of progress has been made in the use of variational methods for investigating homoclinic solutions for Lagrangian systems. Some basic material on variational methods can be found, e.g., in [2,9-11,15,21,22]. As homoclinics are global in time, global methods seem to be the natural approach to study their existence. Both minimization and minimax arguments have been used to obtain them (see $[5,7,8,14,16,18,23]$ ).

The variational formulation for Lagrangian systems usually leads to action functionals. However, even though there may be a natural class of curves or functions as domain of the functional, there is not always a canonical choice of an associated norm or metric. Consequently, it is often challenging to find a good setting in which to formulate the variational problem. To study homoclinic solutions of the problem $(L S)$, a technical framework will be introduced in Sect. 2 to treat the action functional in an appropriate Orlicz-Sobolev space. Section 3 contains the proof of our main result. Let us emphasize that the idea of the proof of Theorem 1.2 differs from [28] as we do not obtain the homoclinic solution as limit of periodic solutions on a finite time interval. Instead we find a candidate for a solution as weak limit of a minimizing sequence and show directly that it is a critical point of the action functional. In Sect. 4 we make final remarks on possible generalisations and future research.

\section{Preliminaries}

Section 2 introduces an appropriate Orlicz-Sobolev space setting and presents some preliminary results about an action functional. The Lemmas 2.4 and 2.5 are similar to Lemmas 2.3 and 2.7 of [17] for the case $N=2$. For the convenience of the reader we include their proofs, thus making our work self-contained.

In what follows, we assume that $\Phi: \mathbb{R}^{N} \rightarrow[0, \infty)$ satisfies $(G 1)-(G 5)$ and $\left(\Delta_{2}\right)$. We define for an open interval $\Omega \subset \mathbb{R}$

$$
L_{\Phi}(\Omega)=\left\{u: \Omega \rightarrow \mathbb{R}^{N}: u \text { is Lebesgue measurable and } \int_{\Omega} \Phi(u(t)) d t<\infty\right\},
$$

which is a Banach space with respect to the Luxemburg norm (cf. [29])

$$
\|u\|_{\Phi}=\inf \left\{v>0: \int_{\Omega} \Phi\left(\frac{u(t)}{v}\right) d t \leq 1\right\} .
$$

Since $\Phi$ is $\Delta_{2}$-regular, $L_{\Phi}(\Omega)$ is separable and reflexive (cf. Rem. 8.22 and Thm. 8.20 in [4]).

Set $\psi=\varphi \circ|\cdot|$, i.e. $\psi(x)=\varphi(|x|)$ for each $x \in \mathbb{R}^{N}$. It follows from (6) that the space $L_{\Phi}(\Omega)$ is continuously embedded in $L_{\psi}(\Omega)$ (cf. Thm. 8.12 in [4]),

$$
L_{\Phi}(\Omega) \subset L_{\psi}(\Omega) .
$$

Note that $\|u\|_{\psi}=\||u|\|_{\varphi}$ and let us recall that Hölder's inequality (cf. [3], Par. 8.11) states that

$$
\int_{\Omega}|u v| d t \leq 2\|u\|_{\psi}\|v\|_{\psi^{*}}, \quad u \in L_{\psi}(\Omega), v \in L_{\psi^{*}}(\Omega) .
$$

Moreover, it is readily seen that

$$
\||u|\|_{\varphi} \leq 1+\int_{\Omega} \varphi(|u(t)|) d t, \quad u \in L_{\psi}(\Omega) .
$$


For simplicity of notation, we abbreviate $L_{\Phi}(\mathbb{R})$ by $L_{\Phi}$. Moreover, even though (7) depends on the domain $\Omega$, we write $\|\cdot\|_{\Phi}$ for the norms of $L_{\Phi}(\Omega)$ for different intervals $\Omega \subset \mathbb{R}$. The domain $\Omega$ will always be clear from the context. In what follows, we will use without further reference that on $\Omega=(a, b)$

$$
\|1\|_{\varphi^{*}}=\left[\left(\varphi^{*}\right)^{-1}\left(\frac{1}{b-a}\right)\right]^{-1},
$$

which follows directly from the definition.

Let $L^{2}\left(\mathbb{R}, \mathbb{R}^{N}\right)$ be the standard Lebesgue space of square integrable functions and let $E$ denote the (mixed) Orlicz-Sobolev space

$$
E=\left\{u \in L^{2}\left(\mathbb{R}, \mathbb{R}^{N}\right): \dot{u} \in L_{\Phi}\left(\mathbb{R}, \mathbb{R}^{N}\right)\right\},
$$

where $\dot{u}$ is the weak derivative of $u$, with the norm

$$
\|u\|^{2}=\|u\|_{2}^{2}+\|\dot{u}\|_{\Phi}^{2}
$$

We note for later reference that $E$ is a separable reflexive Banach space (see [26]).

As usual $L^{\infty}\left(\mathbb{R}, \mathbb{R}^{N}\right)$ denotes the space of Lebesgue measurable essentially bounded functions from $\mathbb{R}$ into $\mathbb{R}^{N}$ with the norm

$$
\|u\|_{\infty}=\operatorname{ess} \sup |u(t)|
$$

and $C\left(\mathbb{R}, \mathbb{R}^{N}\right)$ is the space of continuous functions.

Let us note that if $u \in E$, then $u$ is locally absolutely continuous.

Proposition 2.1 The inclusion map $E \rightarrow L^{\infty}\left(\mathbb{R}, \mathbb{R}^{N}\right) \cap C\left(\mathbb{R}, \mathbb{R}^{N}\right)$ is continuous, i.e. there is a constant $C_{\Phi}>0$ such that for all $u \in E$

$$
\max _{t \in \mathbb{R}}|u(t)|=\|u\|_{\infty} \leq C_{\Phi}\|u\| .
$$

Proof Since

$$
|u(t)| \leq|u(\tau)|+\left|\int_{\tau}^{t} \dot{u}(s) d s\right|
$$

the claimed inequality follows from

$$
\begin{aligned}
|u(t)| & \leq \int_{t-\frac{1}{2}}^{t+\frac{1}{2}}\left(|u(\tau)|+\left|\int_{\tau}^{t} \dot{u}(s) d s\right|\right) d \tau \\
& \leq\left[\int_{t-\frac{1}{2}}^{t+\frac{1}{2}}\left(|u(\tau)|+\left|\int_{\tau}^{t} \dot{u}(s) d s\right|\right)^{2} d \tau\right]^{\frac{1}{2}} \\
& \leq\left[2 \int_{t-\frac{1}{2}}^{t+\frac{1}{2}}\left(|u(\tau)|^{2}+\left|\int_{\tau}^{t} \dot{u}(s) d s\right|^{2}\right) d \tau\right]^{\frac{1}{2}} \\
& \leq\left[2 \int_{t-\frac{1}{2}}^{t+\frac{1}{2}}\left(|u(\tau)|^{2}+\left(\int_{\tau}^{t}|\dot{u}(s)| d s\right)^{2}\right)^{d \tau}\right]^{\frac{1}{2}} \\
& \leq\left[2 \int_{t-\frac{1}{2}}^{t+\frac{1}{2}}\left(|u(\tau)|^{2}+\left(\int_{t-\frac{1}{2}}^{t+\frac{1}{2}}|\dot{u}(s)| d s\right)^{2}\right)^{\frac{1}{2}} d \tau\right]
\end{aligned}
$$




$$
\begin{aligned}
& \leq\left[2 \int_{t-\frac{1}{2}}^{t+\frac{1}{2}}|u(\tau)|^{2} d \tau+2\left(2\|1\|_{\varphi^{*}}\||\dot{u}|\|_{\varphi}\right)^{2}\right]^{\frac{1}{2}} \\
& \leq\left[2\|u\|_{2}^{2}+8\|1\|_{\varphi^{*}}^{2}\|\dot{u}\|_{\Phi}^{2}\right]^{\frac{1}{2}} \leq\left(2+8\|1\|_{\varphi^{*}}^{2}\right)^{\frac{1}{2}}\|u\|,
\end{aligned}
$$

where we have used (8) for functions restricted to $\Omega=\left[t-\frac{1}{2}, t+\frac{1}{2}\right]$. Note that here $\|\cdot\|_{\varphi}$ denotes the norm of $L_{\varphi}(\Omega)$, but $C_{\Phi}:=\left(2+8\|1\|_{\varphi^{*}}^{2}\right)^{\frac{1}{2}}$ is independent of $t$ as $\|1\|_{\varphi^{*}}$ only depends on the length of the interval $\Omega$.

The following proposition shows that functions in $E$ have the right limiting behaviour for studying homoclinics.

Proposition 2.2 If $u \in E$, then $\lim _{t \rightarrow \pm \infty} u(t)=0$.

Proof As in the proof of Proposition 2.1, we obtain

$$
|u(t)| \leq 2\left[\int_{t-\frac{1}{2}}^{t+\frac{1}{2}}|u(\tau)|^{2} d \tau+\left(\int_{t-\frac{1}{2}}^{t+\frac{1}{2}}|\dot{u}(s)| d s\right)^{2}\right]^{\frac{1}{2}} .
$$

Since $u \in L^{2}\left(\mathbb{R}, \mathbb{R}^{N}\right)$, one has

$$
\lim _{t \rightarrow \pm \infty} \int_{t-\frac{1}{2}}^{t+\frac{1}{2}}|u(\tau)|^{2} d \tau=0 .
$$

Furthermore, if $\dot{u} \in L_{\Phi}$ then $|\dot{u}| \in L_{\varphi}$ and so $\int_{\mathbb{R}} \varphi(|\dot{u}|)<\infty$. Consequently, as $\varphi$ is convex, we obtain by Jensen's inequality [26, Prop. III.5]

$$
\lim _{t \rightarrow \pm \infty} \varphi\left(\int_{t-\frac{1}{2}}^{t+\frac{1}{2}}|\dot{u}(s)| d s\right) \leq \lim _{t \rightarrow \pm \infty} \int_{t-\frac{1}{2}}^{t+\frac{1}{2}} \varphi(|\dot{u}(s)|) d s=0,
$$

which finally implies that

$$
\lim _{t \rightarrow \pm \infty} \int_{t-\frac{1}{2}}^{t+\frac{1}{2}}|\dot{u}(s)| d s=0
$$

The following proposition once again considers the embedding of $E$ into $L^{\infty}\left(\mathbb{R}, \mathbb{R}^{N}\right)$.

Proposition 2.3 Every weakly convergent sequence in E contains a subsequence converging in $L_{\text {loc }}^{\infty}\left(\mathbb{R}, \mathbb{R}^{N}\right)$.

Proof Let $\left\{u_{k}\right\}_{k \in \mathbb{N}}$ converge weakly to $u_{0}$ in $E$. By Proposition 2.1, $\left\{u_{k}\right\}_{k \in \mathbb{N}}$ also converges to $u_{0}$ weakly in $L^{\infty}\left(\mathbb{R}, \mathbb{R}^{N}\right)$. Moreover, $\left\|u_{k}\right\| \leq M$ for some $M>0$ and every $k \in \mathbb{N}$.

Let now $a \leq s \leq t \leq b$ for some $a<b$. Then

$$
\begin{aligned}
\left|u_{k}(t)-u_{k}(s)\right| & =\left|\int_{s}^{t} \dot{u}_{k}(\tau) d \tau\right| \leq \int_{s}^{t}\left|\dot{u}_{k}(\tau)\right| d \tau \\
& \leq 2\|1\|_{\varphi_{\Omega}^{*}}\left\|\left|\dot{u}_{k}\right|\right\|_{\varphi_{\Omega}} \leq 2\|1\|_{\varphi_{\Omega}^{*}}\left\|u_{k}\right\| \\
& \leq 2 M\left(\left(\varphi^{*}\right)^{-1}\left(\frac{1}{t-s}\right)\right)^{-1},
\end{aligned}
$$

and thus $\left\{u_{k}\right\}_{k \in \mathbb{N}}$ is a sequence of equicontinuous functions. 
By the Arzelà-Ascoli Theorem, $\left\{u_{k}\right\}_{k \in \mathbb{N}}$ contains a subsequence converging to some $\hat{u}$ in $C\left([a, b], \mathbb{R}^{N}\right)$. It follows from the uniqueness of the weak limit that $\hat{u}=u_{0}$, which completes the proof.

In what follows, let $\Phi: \mathbb{R}^{N} \rightarrow \mathbb{R}$ and $V: \mathbb{R} \times\left(\mathbb{R}^{N} \backslash\{\xi\}\right) \rightarrow \mathbb{R}$ satisfy the assumptions of Theorem 1.2.

We define for $u \in E$

$$
I(u)=\int_{-\infty}^{\infty}(\Phi(\dot{u}(t))-V(t, u(t))) d t .
$$

Let

$$
\alpha_{\varepsilon}=\inf \left\{-V(t, x): x \notin B_{\varepsilon}(0)\right\},
$$

where $0<\varepsilon \leq \frac{1}{2}|\xi|$ and $B_{\varepsilon}(0)$ is the ball of radius $\varepsilon$ centered at the origin. Note that $\alpha_{\varepsilon}>0$ by $\left(V_{1}\right)-\left(V_{3}\right)$.

Proposition 2.4 If $u \in E$ and $u(t) \notin B_{\varepsilon}(0)$ for $t \in[a, b]$, then there is $C_{\varepsilon}>0$ such that

$$
(I(u)+1)^{2} \geq C_{\varepsilon} \cdot \text { length }\left(u_{\mid[a, b]}\right) \geq C_{\varepsilon}|u(b)-u(a)| .
$$

Proof As the second inequality clearly follows from

$$
|u(b)-u(a)|=\left|\int_{a}^{b} \dot{u}(t) d t\right| \leq \int_{a}^{b}|\dot{u}(t)| d t=\text { length }\left(u_{\mid[a, b]}\right),
$$

we only need to prove the first one. We note at first that by (8)

$$
\text { length }\left(u_{\mid[a, b]}\right)=\int_{a}^{b}|\dot{u}(t)| d t \leq 2\||\dot{u}|\|_{\varphi}\|1\|_{\varphi^{*}} .
$$

Hence, if we set $\delta=$ length $\left(u_{\mid[a, b]}\right)$ and $\tau=b-a$, then

$$
\||\dot{u}|\|_{\varphi} \geq \frac{1}{2} \delta\|1\|_{\varphi^{*}}^{-1}=\frac{1}{2} \delta \cdot\left(\varphi^{*}\right)^{-1}\left(\frac{1}{\tau}\right) .
$$

As $\Phi$ is nonnegative, we obtain from (9)

$$
\begin{aligned}
I(u) & \geq \int_{a}^{b}(\Phi(\dot{u}(t))-V(t, u(t))) d t=\int_{a}^{b} \Phi(\dot{u}(t)) d t+\int_{a}^{b}-V(t, u(t)) d t \\
& \geq \int_{a}^{b} \varphi(|\dot{u}(t)|) d t+\alpha_{\varepsilon} \tau \geq\||\dot{u}|\|_{\varphi}-1+\alpha_{\varepsilon} \tau \\
& \geq \frac{1}{2} \delta \cdot\left(\varphi^{*}\right)^{-1}\left(\frac{1}{\tau}\right)-1+\alpha_{\varepsilon} \tau .
\end{aligned}
$$

Thus

$$
I(u)+1 \geq \frac{1}{2} \delta \cdot\left(\varphi^{*}\right)^{-1}\left(\frac{1}{\tau}\right)+\alpha_{\varepsilon} \tau \geq \frac{1}{2} \frac{\delta}{\tau k} \cdot\left(\varphi^{*}\right)^{-1}(k)+\alpha_{\varepsilon} \tau,
$$

where the natural number $k$ satisfies $\tau k \geq 1$ and the last inequality follows from the fact that $\left(\varphi^{*}\right)^{-1}$ is concave. We now choose the smallest $k$ with the property $\tau k \geq 1$. In particular, we set $k=1$ if $\tau \geq 1$. In the latter case,

$$
f(\tau)=\frac{1}{2} \frac{\delta}{\tau} \cdot\left(\varphi^{*}\right)^{-1}(1)+\alpha_{\varepsilon} \tau
$$


has its minimum at the point

$$
\tau_{\min }=\left(\frac{\delta \cdot\left(\varphi^{*}\right)^{-1}(1)}{2 \alpha_{\varepsilon}}\right)^{\frac{1}{2}}
$$

which is equal to $f_{\min }=\left(2 \delta \alpha_{\varepsilon}\left(\varphi^{*}\right)^{-1}(1)\right)^{\frac{1}{2}}$. If $\tau<1$ then

$$
\frac{1}{2} \frac{\delta}{\tau k} \cdot\left(\varphi^{*}\right)^{-1}(k)+\alpha_{\varepsilon} \tau \geq \frac{1}{4} \delta \cdot\left(\varphi^{*}\right)^{-1}(k)+\alpha_{\varepsilon} \tau \geq \frac{1}{4} \delta \cdot\left(\varphi^{*}\right)^{-1}(1) .
$$

Now the claim follows by setting

$$
C_{\varepsilon}=\min \left\{2 \alpha_{\varepsilon}\left(\varphi^{*}\right)^{-1}(1), \frac{1}{4}\left(\varphi^{*}\right)^{-1}(1)\right\} .
$$

In the next proposition, we make use of the Gordon type condition $\left(V_{4}\right)$.

Proposition 2.5 If $[a, b]$ is an interval such that $u([a, b]) \subset \mathcal{N} \backslash\{\xi\}$ then

$$
|U(u(b))|-|U(u(a))| \leq 2(I(u)+1)^{2} .
$$

Proof We first note that by (8),

$$
\begin{aligned}
|U(u(b))| & \leq|U(u(a))|+\left|\int_{a}^{b} \frac{d}{d t} U(u(t)) d t\right| \\
& \leq|U(u(a))|+\left|\int_{a}^{b}(\nabla U(u(t)), \dot{u}(t)) d t\right| \\
& \leq|U(u(a))|+\int_{a}^{b}|\nabla U(u(t)) \| \dot{u}(t)| d t \\
& \leq|U(u(a))|+2\||\nabla U(u)|\|_{\varphi^{*}}\||\dot{u}|\|_{\varphi} .
\end{aligned}
$$

Since by $\left(V_{4}\right)$,

$$
\||\nabla U(u)|\|_{\varphi^{*}} \leq 1+\int_{a}^{b} \varphi^{*}(|\nabla U(u(t))|) d t \leq 1+\int_{a}^{b}-V(t, u(t)) d t,
$$

it is now readily seen from (6) and (9) that

$$
|U(u(b))| \leq|U(u(a))|+2(I(u)+1)^{2} .
$$

It is clear from (14) that $u(t) \neq \xi$ for $t \in \mathbb{R}$ provided that $I(u)<\infty$ (cf. [23], Eq. (2.21)). In fact, we obtain the following corollary.

Corollary 2.6 (cf. [16]) If the action functional I is bounded on a certain set $W \subset E$, say $I(W) \subset[0, \beta]$, then there is $\rho>0$ depending on $\beta$ such that for every $u \in W$ and $t \in \mathbb{R}$ one has $|u(t)-\xi| \geq \rho$.

Our next aim is to show that preimages of bounded sets under $I$ are bounded, which is crucial for our proof of Theorem 1.2.

Proposition 2.7 For every $\beta>0$ the set $I^{-1}([0, \beta])$ is bounded in $E$. 
Proof If $I(u) \leq \beta$, then

$$
\int_{\mathbb{R}} \Phi(\dot{u}(t)) d t \leq \beta \text { and } \int_{\mathbb{R}}-V(t, u(t)) d t \leq \beta .
$$

Hence, in particular $\|\dot{u}\|_{\Phi} \leq \beta+1$, and it remains to find a bound for $\|u\|_{2}$. By $\left(V_{1}\right)$ and $\left(V_{2}\right)$ there is $\gamma>0$ such that

$$
-V^{\prime \prime}(t, 0)(v, v) \geq 2 \gamma|v|^{2}
$$

for $t \in \mathbb{R}$ and $v \in \mathbb{R}^{N}$. Since $V^{\prime \prime}$ is continuous there exists $\delta>0$ such that

$$
-V(t, v) \geq \gamma|v|^{2}
$$

whenever $|v| \leq \delta$ and $t \in \mathbb{R}$. Set

$$
P_{\delta}=\{t \in \mathbb{R}:|u(t)|>\delta\} \text { and } Q_{\delta}=\{t \in \mathbb{R}:|u(t)| \leq \delta\} .
$$

From the inequality

$$
\begin{aligned}
\beta & \geq \int_{P_{\delta}}-V(t, u(t)) d t+\int_{Q_{\delta}}-V(t, u(t)) d t \\
& \geq \alpha_{\delta} \cdot \mu\left(P_{\delta}\right)+\gamma \int_{Q_{\delta}}|u(t)|^{2} d t,
\end{aligned}
$$

we conclude that

$$
\mu\left(P_{\delta}\right) \leq \frac{\beta}{\alpha_{\delta}} \text { and } \int_{Q_{\delta}}|u(t)|^{2} d t \leq \frac{\beta}{\gamma} .
$$

Here $\alpha_{\delta}$ is defined by (11) and $\mu$ denotes the Lebesgue measure. By Proposition 2.4

$$
(I(u)+1)^{2} \geq C_{\delta}\left(2\|u\|_{\infty}-2 \delta\right),
$$

and thus

$$
\|u\|_{\infty} \leq \frac{(I(u)+1)^{2}}{2 C_{\delta}}+\delta \leq \frac{(\beta+1)^{2}}{2 C_{\delta}}+\delta .
$$

Hence we finally obtain

$$
\|u\|_{2}^{2}=\int_{\mathbb{R}}|u(t)|^{2} d t=\int_{P_{\delta}}|u(t)|^{2} d t+\int_{Q_{\delta}}|u(t)|^{2} d t \leq\left(\frac{(\beta+1)^{2}}{2 C_{\delta}}+\delta\right) \frac{\beta}{\alpha_{\delta}}+\frac{\beta}{\gamma} .
$$

We now consider the open set

$$
\Lambda=\left\{u \in E: u(\mathbb{R}) \subset \mathbb{R}^{N} \backslash\{\xi\}\right\} \subset E,
$$

and note that $u \in \Lambda$ iff $I(u)<\infty$ by Corollary 2.6.

We claim that the restricted functional $I: \Lambda \rightarrow \mathbb{R}$ is continuously differentiable. We begin with some auxiliary results.

Lemma 2.8 Let $\left\{u_{n}\right\}_{n=1}^{\infty} \subset L_{\Phi}$ and $u \in L_{\Phi}$. Suppose that $u_{n} \rightarrow u$ a.e. and $\int_{\mathbb{R}} \Phi\left(u_{n}\right) d t \rightarrow$ $\int_{\mathbb{R}} \Phi(u) d t$. Then $u_{n} \rightarrow u$ in $L_{\Phi}$. 
Proof Since $\Phi$ is convex and symmetric, one has

$$
\frac{1}{2}\left(\Phi\left(u_{n}(t)\right)+\Phi(u(t))\right)-\Phi\left(\frac{u_{n}(t)-u(t)}{2}\right) \geq 0
$$

By our assumptions, the right hand side of the above inequality converges to $\Phi(u)$ a.e. Applying Fatou's Lemma we obtain,

$$
\begin{aligned}
\int_{\mathbb{R}} \Phi(u(t)) d t \leq \liminf _{n \rightarrow \infty} \int_{\mathbb{R}}\left[\frac{1}{2}\left(\Phi\left(u_{n}(t)\right)+\Phi(u(t))\right)-\Phi\left(\frac{u_{n}(t)-u(t)}{2}\right)\right] d t \\
=\lim _{n \rightarrow \infty} \int_{\mathbb{R}} \frac{1}{2}\left(\Phi\left(u_{n}(t)\right)+\Phi(u(t))\right) d t+\liminf _{n \rightarrow \infty} \int_{\mathbb{R}}-\Phi\left(\frac{u_{n}(t)-u(t)}{2}\right) d t \\
=\int_{\mathbb{R}} \Phi(u(t)) d t-\limsup _{n \rightarrow \infty} \int_{\mathbb{R}} \Phi\left(\frac{u_{n}(t)-u(t)}{2}\right) d t .
\end{aligned}
$$

This implies that

$$
\int_{\mathbb{R}} \Phi\left(\frac{u_{n}(t)-u(t)}{2}\right) d t \rightarrow 0
$$

and $\left\|u_{n}-u\right\|_{\Phi} \rightarrow 0$ since $\Phi$ satisfies $\Delta_{2}$-property.

Let us note that if $u \in L_{\Phi}$ then $\nabla \Phi(u) \in L_{\Phi^{*}}$, which is a consequence of the inequality (5).

Lemma 2.9 The map $L_{\Phi} \ni u \rightarrow \nabla \Phi(u) \in L_{\Phi^{*}}$ is continuous.

Proof If a sequence $\left\{u_{n}\right\}_{n=1}^{\infty}$ converges to some $u$ in $L_{\Phi}$, then

$$
\int_{\mathbb{R}} \Phi\left(u_{n}(t)-u(t)\right) d t \rightarrow 0 .
$$

Furthermore, $\left\{u_{n}\right\}_{n=1}^{\infty}$ contains a subsequence converging to $u$ a.e. on $\mathbb{R}$. We keep denoting this subsequence by $\left\{u_{n}\right\}_{n=1}^{\infty}$. Consequently $\Phi^{*}\left(\nabla \Phi\left(u_{n}\right)\right) \rightarrow \Phi^{*}(\nabla \Phi(u))$ a.e. on $\mathbb{R}$. By the $\Delta_{2}$-property and the convexity of $\Phi$,

$\Phi\left(2 u_{n}\right)=\Phi\left(2 u+2 u_{n}-2 u\right) \leq \frac{L}{2} \Phi(2 u)+\frac{L}{2} \Phi\left(2 u_{n}-2 u\right) \leq \frac{L^{2}}{2} \Phi(u)+\frac{L^{2}}{2} \Phi\left(u_{n}-u\right)$

and therefore

$$
\begin{aligned}
& \left|\Phi^{*}\left(\nabla \Phi\left(u_{n}\right)\right)-\Phi^{*}(\nabla \Phi(u))\right| \leq \Phi\left(2 u_{n}\right)+\Phi(2 u) \leq\left(\frac{L^{2}}{2}+L\right) \Phi(u)+\frac{L^{2}}{2} \Phi\left(u_{n}-u\right) \\
& \quad \leq L^{\prime}\left(\Phi(u)+\Phi\left(u_{n}-u\right)\right),
\end{aligned}
$$

where $L^{\prime}=\frac{L^{2}}{2}+L$. Applying Fatou's Lemma to the sequence

$$
L^{\prime}\left(\Phi(u)+\Phi\left(u_{n}-u\right)\right)-\left|\Phi^{*}\left(\nabla \Phi\left(u_{n}\right)\right)-\Phi^{*}(\nabla \Phi(u))\right|,
$$

we obtain

$$
\begin{aligned}
& \int_{\mathbb{R}} L^{\prime} \Phi(u) d t \\
& \quad \leq \liminf _{n \rightarrow \infty} \int_{\mathbb{R}}\left[L^{\prime}\left(\Phi(u)+\Phi\left(u_{n}-u\right)\right)-\left|\Phi^{*}\left(\nabla \Phi\left(u_{n}\right)\right)-\Phi^{*}(\nabla \Phi(u))\right|\right] d t
\end{aligned}
$$




$$
\begin{aligned}
& =\lim _{n \rightarrow \infty} \int_{\mathbb{R}} L^{\prime}\left(\Phi(u)+\Phi\left(u_{n}-u\right)\right) d t+\liminf _{n \rightarrow \infty} \int_{\mathbb{R}}-\left|\Phi^{*}\left(\nabla \Phi\left(u_{n}\right)\right)-\Phi^{*}(\nabla \Phi(u))\right| d t \\
& =\int_{\mathbb{R}} L^{\prime} \Phi(u) d t-\limsup _{n \rightarrow \infty} \int_{\mathbb{R}}\left|\Phi^{*}\left(\nabla \Phi\left(u_{n}\right)\right)-\Phi^{*}(\nabla \Phi(u))\right| d t .
\end{aligned}
$$

Consequently,

$$
\lim _{n \rightarrow \infty} \int_{\mathbb{R}}\left|\Phi^{*}\left(\nabla \Phi\left(u_{n}\right)\right)-\Phi^{*}(\nabla \Phi(u))\right| d t=0 .
$$

We finally get the assertion if we apply Lemma 2.8 to the sequence $\left\{\nabla \Phi\left(u_{n}\right)\right\}_{n=1}^{\infty}$

Let us now denote

$$
\mathcal{I}(u)=\int_{\mathbb{R}} \Phi(\dot{u}(t)) d t \text { and } \mathcal{J}(u)=\int_{\mathbb{R}}-V(t, u(t)) d t,
$$

so that $I(u)=\mathcal{I}(u)+\mathcal{J}(u)$.

Proposition $2.10 \mathcal{I} \in C^{1}(E, \mathbb{R})$, and

$$
\mathcal{I}^{\prime}(u) \psi=\int_{\mathbb{R}} \nabla \Phi(\dot{u}(t)) \dot{\psi}(t) d t .
$$

Proof Obviously, if $u \in E$, then $\mathcal{I}(u)<\infty$. It is enough to show that $\mathcal{I}$ has at every point $u \in E$ a directional derivative $\mathcal{I}^{\prime}(u) \in E^{*}$ given by (15) and that the map $\mathcal{I}^{\prime}: E \rightarrow E^{*}$ is continuous. Since $|(\nabla \Phi(x), y)| \leq \Phi(x+y)+\Phi(x)$ for every $x, y \in \mathbb{R}^{N}$, which is a consequence of (3), one has

$$
\begin{aligned}
& \int_{\mathbb{R}}|\nabla \Phi(\dot{u}(t)+s \dot{\psi}(t)) \dot{\psi}(t)| d t \leq \int_{\mathbb{R}} \Phi(\dot{u}(t)+(s+1) \dot{\psi}(t)) d t \\
& +\int_{\mathbb{R}} \Phi(\dot{u}(t)+s \dot{\psi}(t)) d t<\infty .
\end{aligned}
$$

Thus $\mathcal{I}$ has a directional derivative and

$$
\mathcal{I}^{\prime}(u) \psi=\left.\frac{d}{d s} \mathcal{I}(u+s \psi)\right|_{s=0}=\int_{\mathbb{R}} \nabla \Phi(\dot{u}(t)) \dot{\psi}(t) d t .
$$

It follows from Hölder's inequality (8) that

$$
\left|\mathcal{I}^{\prime}(u) \psi\right|=\left|\int_{\mathbb{R}} \nabla \Phi(\dot{u}(t)) \dot{\psi}(t) d t\right| \leq 2\|\nabla \Phi(\dot{u})\|_{L_{\Phi^{*}}}\|\dot{\psi}\|_{L_{\Phi}} \leq C\|\psi\| .
$$

Now it is enough to show that if $u_{n} \rightarrow u$ in $E$, then $\mathcal{I}^{\prime}\left(u_{n}\right) \rightarrow \mathcal{I}^{\prime}(u)$ in $E^{*}$. This is a consequence of the estimate

$$
\begin{aligned}
& \left|\mathcal{I}^{\prime}\left(u_{n}\right) \psi-\mathcal{I}^{\prime}(u) \psi\right|=\left|\int_{\mathbb{R}}\left(\nabla \Phi\left(\dot{u}_{n}(t)\right)-\nabla \Phi(\dot{u}(t))\right) \dot{\psi}(t) d t\right| \\
& \quad \leq 2\left\|\nabla \Phi\left(\dot{u}_{n}(t)\right)-\nabla \Phi(\dot{u}(t))\right\|_{L_{\Phi^{*}}}\|\dot{\psi}(t)\|_{L_{\Phi}} \rightarrow 0,
\end{aligned}
$$

which follows from Lemma 2.9 and Hölder's inequality.

To obtain the differentiability of $\mathcal{J}$, we need to restrict the domain to the open subset $\Lambda$ of $E$. We skip the proof of the following proposition as it is a standard argument. 
Proposition $2.11 \mathcal{J} \in C^{1}(\Lambda, \mathbb{R})$, and

$$
\mathcal{J}^{\prime}(u) \psi=\int_{\mathbb{R}} \nabla V(t, u(t)) \psi(t) d t .
$$

We summarize for later reference the statements of the previous two propositions.

Corollary 2.12 The functional $I: \Lambda \rightarrow \mathbb{R}$ is continuously differentiable.

Each function $u \in \Lambda$ describes a closed curve in $\mathbb{R}^{N} \backslash\{\xi\}$ that starts and ends at 0 . We will consider $\left(\Lambda, \omega_{0}\right)$ as a pointed space with the constant map $\omega_{0}=0$ as distinguished point.

Let $\kappa:(-1,1) \rightarrow \mathbb{R}$ be the homeomorphism defined by the formula

$$
\kappa(t)=\frac{t}{\sqrt{1-t^{2}}} .
$$

If $\sigma \in \Lambda$ then $\hat{\sigma}:[-1,1] \rightarrow \mathbb{R}^{N} \backslash\{\xi\}$

$$
\hat{\sigma}(t)= \begin{cases}\sigma(\kappa(t)) & \text { if }-1<t<1, \\ 0 & \text { if } t \in\{-1,1\},\end{cases}
$$

is a loop at the origin. Let us view the pointed $(N-1)$-sphere $\left(S^{N-1}, \star\right)$ as the quotient space

$$
\pi: S^{N-2} \times[-1,1] \rightarrow S^{N-2} \times[-1,1] /\left(S^{N-2} \times\{-1,1\} \cup\{\star\} \times[-1,1]\right)
$$

where $\pi$ is the corresponding quotient map.

Given a continuous map $h:\left(S^{N-2}, \star\right) \rightarrow\left(\Lambda, \omega_{0}\right)$ we define $\hat{h}:\left(S^{N-1}, \star\right) \rightarrow$ $\left(\mathbb{R}^{N} \backslash\{\xi\},\{0\}\right)$ to be

$$
\hat{h}(\pi(x, t))=\widehat{h(x)}(t) .
$$

This correspondence preserves homotopy classes and induces an isomorphism of homotopy groups

$$
H: \Pi_{N-2}\left(\Lambda, \omega_{0}\right) \rightarrow \Pi_{N-1}\left(\mathbb{R}^{N} \backslash\{\xi\},\{0\}\right) .
$$

Consequently, $\Pi_{N-2}\left(\Lambda, \omega_{0}\right) \cong \Pi_{N-1}\left(\mathbb{R}^{N} \backslash\{\xi\},\{0\}\right) \cong \mathbb{Z}$. Let $\Gamma$ be an element of $\Pi_{N-2}\left(\Lambda, \omega_{0}\right)$. We will write $h \in \Gamma$ if $h:\left(S^{N-2}, \star\right) \rightarrow\left(\Lambda, \omega_{0}\right)$ represents the homotopy class $\Gamma$.

Define

$$
c=\inf _{h \in \Gamma \neq 0} \max _{x \in S^{N-2}} I(h(x)),
$$

which is a nonnegative number.

Definition 2.1 A sequence $\left\{u_{n}\right\}_{n=1}^{\infty} \subset E$ is called a Palais-Smale sequence at level $c\left((P S)_{c}\right.$ for short) if

$$
\lim _{n \rightarrow \infty} I\left(u_{n}\right)=c \quad \text { and } \quad \lim _{n \rightarrow \infty} I^{\prime}\left(u_{n}\right)=0 .
$$

The function $I$ is said to satisfy $(P S)_{c}$ if all $(P S)_{c}$ sequences are compact.

It is a consequence of the General Minimax Principle (see Thm. 1.8 in [1]) that any minimizing sequence for $(17)$ is a $(P S)_{c}$ sequence. 


\section{Proof of Theorem 1.2}

Let $\left\{h_{n}:\left(S^{N-2}, \star\right) \rightarrow\left(\Lambda, \omega_{0}\right)\right\} n=1,2, \ldots$ be a sequence of maps representing nonzero elements of $\Pi_{N-2}\left(\Lambda, \omega_{0}\right)$ and let $I \circ h_{n}: S^{N-2} \rightarrow \mathbb{R}$ achieves its maximum at $x_{n}$. Set $u_{n}=h_{n}\left(x_{n}\right)$ and suppose that $\left\{u_{n}\right\}_{n=1}^{\infty}$ is a minimizing sequence for (17). We now show Theorem 1.2 in five steps.

Step $1 c>0$.

We can assume with no loss of generality that

$$
c \leq I\left(u_{n}\right) \leq c+1, \quad n \in \mathbb{N} .
$$

Since $h_{n} \in \Gamma \neq 0$, there are $q_{n} \in h_{n}\left(S^{N-2}\right)$ and numbers $v_{n}$ as well as $\theta_{n}>1$ such that $q_{n}\left(v_{n}\right)=\theta_{n} \cdot \xi$. In particular,

$$
\left\|q_{n}\right\|_{\infty}>|\xi| .
$$

Moreover, there are $\sigma_{n}, \mu_{n}$ and $\tau_{n} \in\left[\sigma_{n}, \mu_{n}\right]$ such that:

a. $q_{n}\left(\left[\sigma_{n}, \mu_{n}\right]\right) \subset \mathbb{R}^{N} \backslash B_{\frac{|\xi|}{2}}(0)$,

b. $\left|q_{n}\left(\sigma_{n}\right)\right|=\left|q_{n}\left(\mu_{n}\right)\right|=\frac{1}{2}|\xi|$,

c. $\left|q_{n}\left(\tau_{n}\right)\right|=\left\|q_{n}\right\|_{\infty}$

As $I\left(u_{n}\right) \geq I\left(q_{n}\right)$, we obtain from (13),

$$
c+2 \geq I\left(q_{n}\right)+1 \geq \frac{1}{2} \delta \cdot\left(\varphi^{*}\right)^{-1}\left(\frac{1}{\tau}\right)+\alpha_{\varepsilon} \tau
$$

with $\varepsilon=\frac{1}{2}|\xi|$ and $\delta \geq|\xi|$. Hence there are $M>m>0$ such that $m<\tau<M$.

In particular, $\mu_{n}-\sigma_{n}>m$ for each $n \in \mathbb{N}$. Consequently,

$$
c=\inf \left\{I\left(u_{n}\right) ; n \in \mathbb{N}\right\} \geq \inf \left\{I\left(q_{n}\right) ; n \in \mathbb{N}\right\} \geq \alpha_{\varepsilon} m>0 .
$$

Step 2 A minimizing sequence for (17) is bounded in E.

This is an immediate consequence of (18) and Proposition 2.7.

Step $3\left\{u_{n}\right\}_{n=1}^{\infty}$ admits a nonzero weak limit $Q \in \Lambda$.

Since $E$ is reflexive, the sequence $\left\{u_{n}\right\}_{n=1}^{\infty}$ has a subsequence that converges weakly to some $Q \in E$. With no loss of generality we denote this subsequence by $\left\{u_{n}\right\}_{n=1}^{\infty}$, and assume by Proposition 2.3 that $\left\{u_{n}\right\}_{n=1}^{\infty}$ converges strongly in $L_{\text {loc }}^{\infty}\left(\mathbb{R}, \mathbb{R}^{N}\right)$.

It follows from Fatou's Lemma that $I(Q) \leq c$. Thus $Q \in \Lambda$ by Corollary 2.6.

We now want to prove that $Q \neq \omega_{0}$. Let $h:\left(S^{N-2}, \star\right) \rightarrow\left(\Lambda, \omega_{0}\right)$ be homotopically nontrivial and let $\max (I \circ h)<s \cdot c$ for some $s>1$. Consider $h_{0}:\left(S^{N-2}, \star\right) \rightarrow\left(\Lambda, \omega_{0}\right)$,

$$
h_{0}(x)=\left\{\begin{array}{lll}
\frac{\|h(x)\|_{\infty}}{\delta} h(x) & \text { if } & \|h(x)\|_{\infty} \leq \delta \\
h(x) & \text { if } & \|h(x)\|_{\infty}>\delta
\end{array}\right.
$$

where $\delta$ is the constant from $\left(V_{*}\right)$. Obviously, $h_{0}$ is homotopic to $h$.

If $h(x)=\sigma$ and $\|\sigma\|_{\infty}=\frac{\delta}{s}$ then by $\left(V_{*}\right)$

$$
I\left(h_{0}(x)\right)=\int_{-\infty}^{\infty}\left(\Phi\left(\frac{1}{s} \dot{\sigma}(t)\right)-V\left(t, \frac{1}{s} \sigma(t)\right)\right) d t \leq \frac{1}{s} \cdot I(h(x))<c .
$$

Thus, $I \circ h_{0}$ achieves its maximum at some $x_{0} \in S^{N-2}$ such that $\left\|h_{0}\left(x_{0}\right)\right\|_{\infty}>\frac{\delta}{s}$. Let us note that $I\left(h_{0}(x)\right) \leq I(h(x))$ for every $x \in S^{N-2}$. 
Consequently, we can assume that the minimizing sequence $\left\{u_{n}\right\}_{n=1}^{\infty}$ for (17), i.e. $u_{n}=$ $h_{n}\left(x_{n}\right), I\left(h_{n}\left(x_{n}\right)\right)<s_{n} \cdot c, s_{n}>1$ and $\lim _{n \rightarrow \infty} s_{n}=1$, satisfies $\left\|h_{n}\left(x_{n}\right)\right\|_{\infty}>\frac{\delta}{s_{n}}$ for every $n \in \mathbb{N}$.

For a later step of the proof, let us slightly modify the sequence $\left\{u_{n}\right\}_{n=1}^{\infty}$. Let $\gamma_{k}: \Lambda \rightarrow \Lambda$ denote the shift $\gamma_{k} \sigma(t)=\sigma(t+k)$. Since the potential $V$ is 1-periodic one has $I\left(\gamma_{k} \sigma\right)=I(\sigma)$ for $k \in \mathbb{Z}$. Every $\sigma \in \Lambda$ can be shifted in such a way that its sup-norm is achieved on the interval $[0,1]$. Therefore, we can suppose that every element of the minimizing sequence $\left\{u_{n}\right\}_{n=1}^{\infty}$ has this property.

Since $Q$ is the weak limit of $\left\{u_{n}\right\}_{n=1}^{\infty}$ in $E$, it is also the limit in $L_{l o c}^{\infty}\left(\mathbb{R}, \mathbb{R}^{N}\right)$ and thus $\|Q\|_{\infty} \geq \delta$.

Step $4 I^{\prime}(Q)=0$.

It suffices to prove that for any $v \in C_{0}^{\infty}\left(\mathbb{R}, \mathbb{R}^{N}\right)$,

$$
I^{\prime}(Q)[v]=\int_{-\infty}^{\infty}\left(\nabla \Phi(\dot{Q}(t)) \dot{v}(t)-\nabla V_{u}(t, Q(t)) v(t)\right) d t=0 .
$$

Let $T>0$ be such that $\operatorname{supp}(v) \subset[-T, T]$. Let us recall from Step 3 that our $(P S)_{c}$ sequence $\left\{u_{n}\right\}_{n=1}^{\infty}$ has the nonzero weak limit $Q \in \Lambda$. As the modulus function increases essentially slower than $\Phi$ near infinity, the sequence $\left\{\dot{u}_{n}\right\}_{n=1}^{\infty}$ converges to $\dot{Q}$ strongly in $L_{l o c}^{1}\left(\mathbb{R}, \mathbb{R}^{N}\right)$. In particular, $\left\{\dot{u}_{n}\right\}_{n=1}^{\infty}$ converges to $\dot{Q}$ strongly in $L^{1}\left([-T, T], \mathbb{R}^{N}\right)$ and thus contains a subsequence converging to $\dot{Q}$ almost everywhere on $[-T, T]$. With no loss of generality we keep denoting this subsequence by $\left\{u_{n}\right\}_{n=1}^{\infty}$. As $\nabla \Phi$ is continuous, the sequence $\left\{\nabla \Phi\left(\dot{u}_{n}\right)\right\}_{n=1}^{\infty}$ converges to $\nabla \Phi(\dot{Q})$ almost everywhere on $[-T, T]$. By (5), it follows that

$$
\int_{\mathbb{R}} \Phi^{*}\left(\nabla \Phi\left(\dot{u}_{n}(t)\right)\right) d t \leq L \int_{\mathbb{R}} \Phi\left(\dot{u}_{n}(t)\right) d t \leq L I\left(u_{n}\right) \leq L(c+1),
$$

where we have used (18) in the last inequality. Consequently, the sequence $\left\{\nabla \Phi\left(\dot{u}_{n}\right)\right\}_{n=1}^{\infty}$ is bounded in $L_{\Phi^{*}}$, and so it contains a subsequence that converges weakly to some $u_{0} \in L_{\Phi^{*}}$. By [6, Thm. 2.3], the modulus function increases essentially slower than $\Phi^{*}$ near infinity, and so we see that the latter sequence actually converges strongly to $u_{0}$ in $L_{l o c}^{1}\left(\mathbb{R}, \mathbb{R}^{N}\right)$. Thus it contains a subsequence converging to $u_{0}$ almost everywhere on $[-T, T]$. As before, we do not change the indices of our sequence for the sake of simplicity of notation. In summary, the sequence $\left\{\nabla \Phi\left(\dot{u}_{n}\right)\right\}_{n=1}^{\infty}$ converges to $\nabla \Phi(\dot{Q})$ almost everywhere on $[-T, T]$ and it also converges to $u_{0}$ almost everywhere on $[-T, T]$. Consequently, $\nabla \Phi(\dot{Q})=u_{0}$ almost everywhere on $[-T, T]$. As $u_{0}$ is the weak limit of $\left\{\nabla \Phi\left(\dot{u}_{n}\right)\right\}_{n=1}^{\infty}$ in $L_{\Phi^{*}}$, we obtain

$$
\begin{gathered}
\int_{\mathbb{R}} \dot{v}(t) \nabla \Phi\left(\dot{u}_{n}(t)\right) d t \stackrel{n \rightarrow \infty}{\longrightarrow} \int_{\mathbb{R}} \dot{v}(t) u_{0}(t) d t=\int_{-T}^{T} \dot{v}(t) u_{0}(t) d t \\
=\int_{-T}^{T} \dot{v}(t) \nabla \Phi(\dot{Q}(t)) d t=\int_{\mathbb{R}} \dot{v}(t) \nabla \Phi(\dot{Q}(t)) d t,
\end{gathered}
$$

where we have used that $\operatorname{supp}(\dot{v}) \subset \operatorname{supp}(v) \subset[-T, T]$. Moreover, as $\left\{u_{n}\right\}_{n=1}^{\infty}$ converges to $Q$ strongly in $L_{\text {loc }}^{\infty}\left(\mathbb{R}, \mathbb{R}^{N}\right.$ ) (see beginning of Step 3), we have

$$
\begin{aligned}
\int_{\mathbb{R}} v(t) \nabla V_{u}\left(t, u_{n}(t)\right) d t \\
\quad=\int_{-T}^{T} v(t) \nabla V_{u}\left(t, u_{n}(t)\right) d t \stackrel{n \rightarrow \infty}{\longrightarrow} \int_{-T}^{T} v(t) \nabla V_{u}(t, Q(t)) d t \\
\quad=\int_{\mathbb{R}} v(t) \nabla V_{u}(t, Q(t)) d t .
\end{aligned}
$$


Finally, we obtain from (20) and (21)

$$
\begin{aligned}
I^{\prime}(Q)[v] & =\int_{\mathbb{R}}\left(\nabla \Phi(\dot{Q}(t)) \dot{v}(t)-\nabla V_{u}(t, Q(t)) v(t)\right) d t \\
& =\lim _{n \rightarrow \infty} \int_{\mathbb{R}}\left(\nabla \Phi\left(\dot{u}_{n}(t)\right) \dot{v}(t)-\nabla V_{u}\left(t, u_{n}(t)\right) v(t)\right) d t \\
& =\lim _{n \rightarrow \infty} I^{\prime}\left(u_{n}\right)[v]=0,
\end{aligned}
$$

where we have used in the last equality that $\left\{u_{n}\right\}_{n=1}^{\infty}$ is a $(P S)_{c}$ sequence.

Step $5 \dot{Q}(t) \rightarrow 0, t \rightarrow \pm \infty$.

In this final step of our proof we show that $Q$ is homoclinic to 0 , i.e. $\dot{Q}(t) \rightarrow 0$ as $t \rightarrow \pm \infty$. Note that we have already shown that $Q(t) \rightarrow 0$ as $t \rightarrow \pm \infty$ since $Q \in \Lambda$ by Step 3. To see that the derivatives have the right limiting behaviour as well, we set for $t \in \mathbb{R}$

$$
x(t):=\nabla \Phi(\dot{Q}(t)) .
$$

Note that $x$ is continuously differentiable, as its weak derivative is the continuous map $-\nabla_{u} V(t, Q(t))$, and

$$
\dot{x}(t)=\frac{d}{d t}(\nabla \Phi(\dot{Q}(t)))=-\nabla_{u} V(t, Q(t)) \rightarrow 0, \quad t \rightarrow \pm \infty .
$$

In what follows we only consider the case $t \rightarrow \infty$ as the necessary modifications for the other limit are obvious. Let $\varepsilon>0$ be given and let $\delta_{1}>0$ be such that if $\Phi^{*}(v)<\delta_{1}$ for $v \in \mathbb{R}^{N}$, then $|v|<\varepsilon$. Let us note that the existence of $\delta_{1}$ follows from the convexity of $\Phi^{*}$ and is a simple exercise in analysis. By (22), there is $R_{1}>0$ such that

$$
|\dot{x}(t)|<\varepsilon, \quad t \geq R_{1} .
$$

As $x=\nabla \Phi(\dot{Q}) \in L_{\Phi^{*}}($ see Step 4),

$$
\int_{\mathbb{R}} \Phi^{*}(x(t)) d t<\infty
$$

and so there is $R_{2}>0$ such that

$$
\int_{R_{2}}^{+\infty} \Phi^{*}(x(t)) d t<\delta_{1} .
$$

We now set $R:=\max \left\{R_{1}, R_{2}\right\}$ and let $t>R$. For $\bar{R}>R$ such that $t \in[\bar{R}, \bar{R}+1]$ we have by (24)

$$
\int_{\bar{R}}^{\bar{R}+1} \Phi^{*}(x(s)) d s<\delta_{1} .
$$

Consequently, by the Mean Value Theorem there is some $t_{0} \in[\bar{R}, \bar{R}+1]$ such that $\Phi^{*}\left(x\left(t_{0}\right)\right)<\delta_{1}$ and so $\left|x\left(t_{0}\right)\right|<\varepsilon$ by our choice of $\delta_{1}$. We obtain from (23)

$$
\left|x(t)-x\left(t_{0}\right)\right|=\left|\int_{t_{0}}^{t} \dot{x}(s) d s\right| \leq \int_{\bar{R}}^{\bar{R}+1}|\dot{x}(s)| d s<\varepsilon
$$

and so

$$
|x(t)|<\left|x\left(t_{0}\right)\right|+\varepsilon<2 \varepsilon .
$$


In summary, we have shown that for every $\varepsilon>0$ there is some $R>0$ such that $|x(t)|<2 \varepsilon$ for $t \geq R$. Consequently, $x(t) \rightarrow 0$ as $t \rightarrow+\infty$, which in turn implies that $\dot{Q}(t) \rightarrow 0$ as $t \rightarrow+\infty$ since 0 is the only critical point of $\Phi$ and $\lim _{x \rightarrow \infty}|\nabla \Phi(x)|=0$.

\section{Final remarks}

The Gordon type condition $\left(V_{4}\right)$ in Theorem 1.2 excludes gravitational potentials. Unfortunately, it is crucial for our argument as it gives a sufficient condition for the existence of nontrivial homotopy groups of the loop space $\Lambda \subset E$. Analogously to the winding number considered by Rabinowitz for $N=2$ (see e.g. [23]), elements of nontrivial homotopy groups are essential for defining minimax values of the action integral $I$ for $N \geq 3$. It is an interesting open problem if other methods might yield Theorem 1.2 under a weaker assumption than $\left(V_{4}\right)$ to include gravitational potentials.

A further interesting problem related to our work is the existence of multibump solutions for $(L S)$. These are solutions near sums of sufficiently separated translates of a basic homoclinic solution, which exists for $(L S)$ by our Theorem 1.2. The only results for singular Hamiltonians we are aware of concern the case $N=2$ (see [24,25] and references therein). It is an interesting question whether a similar approach also works in higher dimensions and Orlicz-Sobolev spaces, which we shall address in a future publication.

We conclude our work by explaining the regularity of solutions of $(L S)$ in case that $\Phi$ is strictly convex. Let us first recall that the map $x(t)=\nabla \Phi(\dot{Q}(t))$ in Step 5 is continuously differentiable. It follows from Corollary 4.1.3 and the formula (0.2) in Section E of [13] that, if $\Phi$ is strictly convex, then $\nabla \Phi: \mathbb{R}^{N} \rightarrow \mathbb{R}^{N}$ is invertible and its inverse map $(\nabla \Phi)^{-1}=\nabla \Phi^{*}$ is continuous. Thus $\dot{Q}(t)=\nabla \Phi^{*}(x(t))$ is continuous and so our solution $Q$ is continuously differentiable. Moreover, if in addition $\nabla \Phi^{*}$ is $C^{1}$, then $\dot{Q}$ is a composition of $C^{1}$ maps and consequently $Q$ is $C^{2}$, i.e., a classical solution. Finally, let us point out that these additional assumptions are in particular satisfied for $\Phi(x)=\frac{|x|^{p}}{p}, 1<p \leq 2$, as in this case $\Phi^{*}(x)=$ $\frac{|x|^{q}}{q}$ for $\frac{1}{p}+\frac{1}{q}=1$, so that $q \geq 2$.

Acknowledgements The research is supported by Grant BEETHOVEN2 of the National Science Centre, Poland, No. 2016/23/G/ST1/04081. The authors wish to thank Luca Asselle for several helpful comments. The authors are also greatly indebted to the referee for some stimulating questions and for drawing our attention to some interesting manuscripts.

Open Access This article is licensed under a Creative Commons Attribution 4.0 International License, which permits use, sharing, adaptation, distribution and reproduction in any medium or format, as long as you give appropriate credit to the original author(s) and the source, provide a link to the Creative Commons licence, and indicate if changes were made. The images or other third party material in this article are included in the article's Creative Commons licence, unless indicated otherwise in a credit line to the material. If material is not included in the article's Creative Commons licence and your intended use is not permitted by statutory regulation or exceeds the permitted use, you will need to obtain permission directly from the copyright holder. To view a copy of this licence, visit http://creativecommons.org/licenses/by/4.0/.

\section{References}

1. Abbondandolo, A.: Lectures on the free period Lagrangian action functional. J. Fixed Point Theory Appl. 13, 397-430 (2013)

2. Ambrosetti, A., Coti Zelati, V.: Periodic Solutions of Singular Lagrangian Systems. Birkhäuser, Basel (1993) 
3. Aubin, J.P.: Optima and Equilibria, Graduate Text in Mathematics, vol. 140. Springer, Berlin (1993)

4. Adams, R.A., Fournier, J.J.F.: Sobolev Spaces, Pure and Applied Mathematics, vol. 140. Academic Press, New York (2009)

5. Byeon, J., Montecchiari, P., Rabinowitz, P.H.: A double well potential system. Anal. PDE 9(7), 1737-1772 (2016)

6. Chmara, M., Maksymiuk, J.: Anisotropic Orlicz-Sobolev spaces of vector valued functions and Lagrange equations. J. Math. Anal. Appl. 456(1), 457-475 (2017)

7. Cieliebak, K., Séré, E.: Pseudoholomorphic curves and multiplicity of homoclinic orbits. Duke Math. J. 77, 483-518 (1995)

8. Coti Zelati, V., Ekeland, I., Séré, E.: A variational approach to homoclinic orbits in Hamiltonian systems. Math. Ann. 288, 133-160 (1990)

9. Coti Zelati, V., Rabinowitz, P.H.: Homoclinic orbits for second order Hamiltonian systems possessing superquadratic potentials. J. Am. Math. Soc. 4, 693-727 (1991)

10. Coti Zelati, V., Montecchiari, P., Nolasco, M.: Multibump homoclinic solutions for a class of second order, almost periodic Hamiltonian systems. NoDEA Nonlinear Differ. Equ. Appl. 4, 77-99 (1997)

11. Ekeland, I.: Convexity Methods in Hamiltonian Mechanics. Springer, Berlin (1990)

12. Gordon, W.B.: Conservative dynamical systems involving strong forces. Trans. Am. Math. Soc. 204, 113-135 (1975)

13. Hiriart-Urruty, J.-B., Lemarechal, C.: Fundamentals of Convex Analysis, Grundlehren Text Editions. Springer, Berlin (2001)

14. Hofer, H., Wysocki, K.: First order elliptic systems and the existence of homoclinic orbits in Hamiltonian systems. Math. Ann. 288, 483-503 (1990)

15. Hofer, H., Zehnder, E.: Hamiltonian Dynamics and Symplectic Invariants. Birkhäuser, Basel (1994)

16. Izydorek, M., Janczewska, J.: The shadowing chain lemma for singular Hamiltonian systems involving strong forces. Cent. Eur. J. Math. 10, 1928-1939 (2012)

17. Izydorek, M., Janczewska, J., Mawhin, J.: Homoclinics for singular strong force Lagrangian systems. Adv. Nonlinear Anal. 9, 644-653 (2020). https://doi.org/10.1515/anona-2020-0018

18. Janczewska, J., Maksymiuk, J.: Homoclinic orbits for a class of singular second order Hamiltonian systems in $\mathbb{R}^{3}$. Cent. Eur. J. Math. 10, 1920-1927 (2012)

19. Krasnosel'skiǔ, M.A., Rutickiǔ, YaB: Convex Functions and Orlicz Spaces. P. Noordhoff Ltd., Groningen (1961)

20. Le, V.K.: On second order elliptic equations and variational inequalities with anisotropic principal operators. Topol. Methods Nonlinear Anal. 44, 41-72 (2014)

21. Mawhin, J., Willem, M.: Critical Point Theory and Hamiltonian Systems, Applied Mathematical Sciences, vol. 74. Springer, New York (1989)

22. Rabinowitz, P.H.: Minimax Methods in Critical Point Theory with Applications to Differential Equations. CBMS Regional Conference Series in Mathematics, vol. 65 (1986)

23. Rabinowitz, P.H.: Homoclinics for a singular Hamiltonian system. In: Jost, J. (ed.) Geometric Analysis and the Calculus of Variations, pp. 267-297. International Press, Cambridge (1996)

24. Rabinowitz, P.H.: Multibump solutions of differential equations: an overview. Chin. J. Math. 24(1), 1-36 (1996)

25. Rabinowitz, P.H.: Variational methods for Hamiltonian systems. In: Hasselblatt, B., Katok, A. (eds.) Handbook of Dynamical Systems, vol. 1A, pp. 1091-1127. Elsevier Science B.V., Amsterdam (2002)

26. Rao, M.M., Ren, Z.D.: Theory of Orlicz Spaces. Marcel Dekker Inc., New York (1991)

27. Rockafellar, R.T.: Convex Analysis. Princeton University Press, Princeton (1970)

28. Tanaka, K.: Homoclinic orbits for a singular second order Hamiltonian system. Ann. Inst. H. Poincaré 7(5), 427-438 (1990)

29. Trudinger, N.S.: An imbedding theorem for $H^{0}(G, \Omega)$ spaces. Stud. Math. 50(1), 17-30 (1974)

Publisher's Note Springer Nature remains neutral with regard to jurisdictional claims in published maps and institutional affiliations. 